\title{
TRAUMA HEPÁTICO: EPIDEMIOLOGIA DE CINCOANOS EM UM SERVIÇO DE EMERGÊNCIA
}

\author{
HEPATIC TRAUMA: FIVE YEARS OF EPIDEMIOLOGY IN AN EMERGENCY \\ DEPARTMENT
}

\author{
Carla Martinez Menini Stalhschmidt, ACBC-PR ${ }^{1}$; Beatriz Formighieri² \\ Débora Majszak Marcon ${ }^{3}$; Aline Luri Takejima ${ }^{3}$; Luis Guilherme Sanches Soares ${ }^{4}$
}

\begin{abstract}
RESUMO: Objetivo: analisar características epidemiológicas relacionadas ao trauma hepático e fazer breve revisão das modalidades diagnósticas e de tratamento. Método: estudo retrospectivo de fevereiro/2002 a maio/2007 através de prontuários de 154 pacientes admitidos com trauma hepático no Hospital Universitário Cajuru (HUC). Resultados: Foram encontrados 90,26\% das vítimas de trauma hepático do sexo masculino e a média de idade de 26,28 anos. Quanto ao mecanismo de trauma, $72,73 \%$ foram por trauma penetrante, sendo que destes, $55,84 \%$ foram por arma de fogo e $16,88 \%$ por arma branca; e $27,27 \%$ por trauma contuso, no qual $73,81 \%$ envolveram colisões por veículos automotores e $26,49 \%$ outros. Na admissão o período de $0 \mathrm{~h}-12 \mathrm{~h}$ foi o de maior prevalência, a média da pressão arterial foi de 117,6/72,3 mmHg, da frequiência cardíaca de 99,03 bpm e do Glasgow de 13,6. O tempo decorrido entre a admissão e a realização da primeira cirurgia foi de menos de 2 horas em 60,43\%. Verificou- se maior incidência da lesão Grau II, seguida da Grau III e IV (totalizando 88,3\%). As lesões cirúrgicas associadas foram encontradas em mais de $75 \%$ dos casos. O ISS médio foi de $15,09,19,85,27,83,35,47$ e 40,93 e a sobrevida de $100 \%, 88,88 \%, 81,25 \%, 48,48 \%$ e $22,23 \%$ nas lesões grau I, II, III, IV e V, respectivamente. Conclusão: os dados epidemiológicos encontrados neste estudo refletem a violência na sociedade moderna, que se traduz com aumento da complexidade das lesões encontradas e constitui desafio para decisão da melhor conduta terapêutica (Rev. Col. Bras. Cir. 2008; 35(4): 225-228).
\end{abstract}

Descritores: Fígado; Traumatismos abdominais; Ferimentos e lesões/epidemiologia.

\section{INTRODUÇÃO}

As primeiras descrições formais do trauma hepático datam antes de 1800, embora haja descrições de tratamento das lesões hepáticas na mitologia grega e romana. A terapêutica dessas lesões modificou-se gradualmente no século 20 , principalmente com as experiências militares ${ }^{1}$. Apesar da sistematização do atendimento ao politraumatizado proposta pelo Advanced Trauma Life Support (ATLS) e da evolução no manejo peri-operatório, tem-se observado que a morbi-mortalidade permanece elevada ${ }^{2}$.

O presente estudo tem por objetivo analisar as características epidemiológicas relacionadas ao trauma hepático e fazer uma breve revisão das modalidades diagnósticas e de tratamento.

\section{MÉTODO}

No período de fevereiro/2002 à maio/2007 foram revistos os prontuários de pacientes atendidos no Hospital Universitário Cajuru de Curitiba com lesão hepática produzida por trauma contuso e penetrante. Os parâmetros analisados foram: idade, sexo, horário de admissão e dados vitais, grau da lesão, horário da primeira operação, presença de lesões associadas e cálculo de ISS, tempo de internamento e evolução para o óbito. Estes dados foram submetidos a análise estatística.

\section{RESULTADOS}

Foram atendidos, no período citado acima, 319.354 pacientes sendo que o trauma hepático ocorreu em 154 (5\%) deles, dos quais 139 eram do sexo masculino $(90,26 \%)$ e 15 do sexo feminino $(9,74 \%)$. A média das idades dos pacientes foi de 26,28 anos, variando de 6 a 70 anos (mediana $=24$ anos). $\mathrm{O}$ trauma penetrante ocorreu em mais de $2 / 3$ dos casos (112), e o principal mecanismo foi por ferimentos por arma de fogo (FAF) e arma branca (FAB), enquanto que o trauma contuso ocorreu em aproximadamente $1 / 3$ dos pacientes (42) e as colisões envolvendo veículos automotores (VAM) foram o agente etiológico em mais de $70 \%$ dos pacientes (figura 1).

$\mathrm{Na}$ admissão dos pacientes no serviço de emergência do hospital o horário foi dividido em três períodos das $0 \mathrm{~h}$ $-12 \mathrm{~h}, 12 \mathrm{~h}-20 \mathrm{~h}, 20 \mathrm{~h}-0 \mathrm{~h}$, representando respectivamente $41,56 \%, 29,22 \%$ e $29,22 \%$. A média da pressão arterial dos pacientes foi de $117,6 / 72,3 \mathrm{mmHg}$, a da freqüência cardíaca de 99,03 bpm e a do Glasgow de 13,6.

O tempo decorrido entre a admissão no hospital e a realização da primeira operação foi menor que duas horas em

1. Cirurgiã do Trauma do Serviço de Cirurgia Geral - Trauma do Hospital Universitário Cajuru, Curitiba - PR; Instrutora do ATLS.

2. Residente da Cirurgia Geral do Hospital Universitário Cajuru.

3. Acadêmica do $6^{\circ}$ ano de Medicina da Pontifícia Universidade Católica do Paraná.

4. Médico graduado pela Pontifícia Universidade Católica do Paraná.

Recebido em 01/02/2008

Aceito para publicação em 02/04/2008

Conflito de interesses: nenhum

Fonte de financiamento: nenhuma

Trabalho realizado no Serviço de Cirurgia Geral - Trauma do Hospital Universitário Cajuru, Curitiba - PR. 


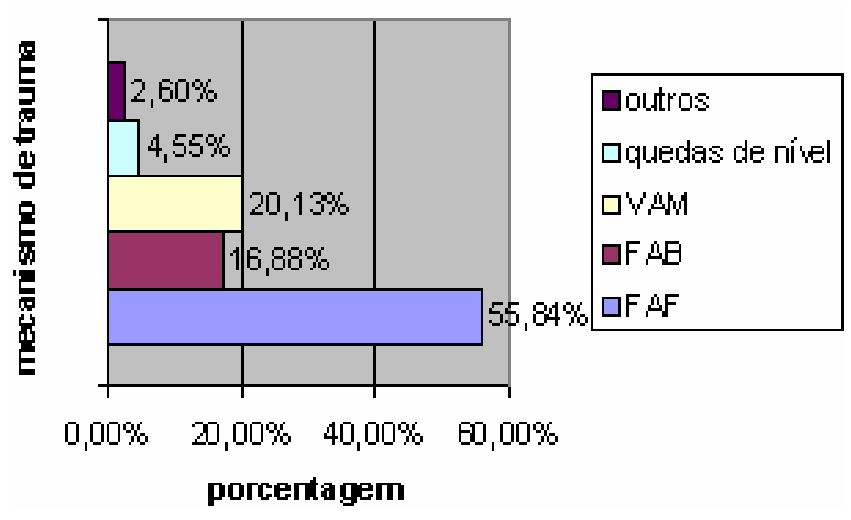

Figura 1 - Mecanismos de trauma hepático.

$60,43 \%$, entre duas e quatro horas em $21,58 \%$ e mais que quatro horas em $17,99 \%$. A maioria dos submetidos ao tratamento cirúrgico com menos de duas horas possuíam lesões Grau IV e V. Houve maior incidência de lesão Grau II, seguida das de Grau III e IV. As lesões leves (até Grau III) corresponderam a $72,71 \%$, como consta no figura 2 .

Em relação à terapêutica a tendência ao tratamento expectante (somente observação da lesão) nos traumas hepáticos foi adotada em ordem decrescente nas lesões Grau I (66,66\%), Grau II (32,69\%), Grau III (4,08\%), Grau IV (3,03\%) e Grau V (0\%).

Nos pacientes com Grau I havia lesões associadas em 77,8\%, com ISS médio de 15,09. No Grau II em 75,93\%, com ISS de 19,85. Já no Grau III ocorreram em $83.67 \%$, com ISS de 27,83. Enquanto que no Grau IV foram em 90,91\%, com ISS de 35,47. E finalmente, nas lesões Grau V todos apresentaram lesões associadas, com ISS médio de 40,93.

A média de dias de internamento foi de 5,44, 7,22, 9,65, 7,94 e 7,11 (mediana $=6$ ) e a sobrevida de $100 \%, 88,88 \%, 81,25 \%$, $48,48 \%$ e 22,23\% nas lesões Grau I, II, III, IV e V, respectivamente.

\section{DISCUSSÃO}

O paciente com trauma hepático corresponde a aproximadamente $5 \%$ das admissões das salas de urgência ${ }^{3}$. O fígado é um dos órgãos intra-abdominais mais acometidos no trauma pelo seu tamanho e sua localização anatômica ${ }^{4,5}$. Os mecanismos de trauma hepático, contuso e penetrante, variam em proporção de acordo com a região. Um estudo na Escócia com 783 pacientes entre 1992 - 2002 mostrou que o mecanismo de trauma contuso ocorreu em $69 \%$ dos casos e penetrante em $31 \%{ }^{6}$. Outros autores europeus encontraram um índice de trauma contuso ainda maior, de $80 \%-90 \%$, na Alemanha e Suécia ${ }^{7,8}$, de $94 \%$ no Reino Unido 9 e $91 \%$ em Estocol-
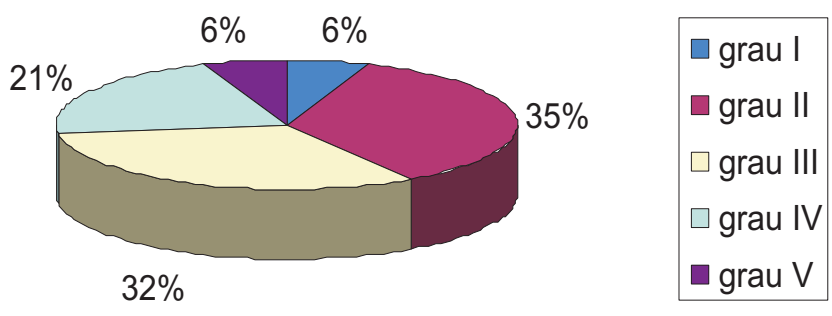

Figura 2 - Grau das lesões hepáticas. $\mathrm{mo}^{10}$. Já na África do Sul $66 \%$ dos traumas foram penetrantes ${ }^{11}$. Na América do Norte o trauma penetrante também foi o mais comum segundo a revisão de Feliciano et al ${ }^{12}$ com $86 \%$ dos 1000 pacientes. Em um Hospital Universitário Universitário de Curitiba - Brasil, no período de 1999 - 2003, encontrouse taxas de $54,6 \%$ de trauma penetrante e $46,4 \%$ de contuso ${ }^{13}$. Portanto nota-se que no continente africano e americano, onde há grande número de países em desenvolvimento, predomina o trauma penetrante, em contraste com os países europeus em que o trauma contuso ocorre em maior proporção.

Atualmente o cenário de violência das grandes e médias cidades, vem demonstrando o aumento da incidência de homicídios incluindo armas semi-automáticas (antigamente apenas de uso militar). Isto se reflete no aumento da complexidade das lesões encontradas nas vítimas, e consequentemente em um novo desafio para todos os cirurgiões. Acrescido a isso, a alta tecnologia empregada nos veículos automotivos, tem aumentado a energia cinética nos traumas contusos, ampliando o espectro de lesões graves e complexas a serem reparadas ${ }^{14}$. Neste estudo os ferimentos por arma de fogo e acidentes envolvendo veículos automotores representaram juntos $82,47 \%$ dos mecanismos de trauma. $\mathrm{O}$ Colégio Americano de Cirurgiões em seu banco de dados de 1994 a 2003 concluiu que dos $78 \%$ de trauma contuso a maioria foi causada por acidentes envolvendo veículos automotores $(72,7 \%)$, seguido de assaltos $(20,3 \%)^{15}$. No Rio Grande do Sul dos 61 pacientes com trauma penetrante $56 \%$ tiveram ferimentos por arma de fogo e $44 \%$ por arma branca ${ }^{2}$.

Em relação ao sexo dos pacientes vítimas de trauma hepático houve prevalência masculina em todos os artigos analisados, sendo de $64,6 \%$ no relato do Colégio Americano de Cirurgiões ${ }^{15}$, e de $76 \%$ conforme Scollay, na Escócia ${ }^{6}$. A média de idade foi de 26,28 anos no presente estudo, 35, 32, 31, 30 anos, na Alemanha ${ }^{16}$, África do Sul ${ }^{11}$, Escócia ${ }^{6}$ e Estados Unidos da América ${ }^{17}$, respectivamente, demonstrando a prevalência na população jovem.

As lesões associadas foram encontradas em 83,12\% dos pacientes, em conformidade com Scollay que encontrou 90\% de lesões intra e extra-abdominais (cabeça, pescoço, ortopédicas). $\mathrm{O}$ alto número de lesões associadas é um importante desafio para a condução dos pacientes pois sua presença dificulta a decisão do tratamento conservador ${ }^{6}$.

AAssociação Americana para cirurgia do trauma estabeleceu um sistema detalhado para classificação das lesões hepáticas utilizado neste trabalho e em toda a literatura mundial ${ }^{18}$, como mostra a tabela 1 .

No trauma de fígado, as lesões leves (até Grau III) correspondem à maioria $(85 \%)$ de todas as lesões hepáticas ${ }^{3,5}$. Neste estudo foram $72,71 \%$. A mortalidade em trauma hepático é alta, atingindo níveis de 35 a $80 \%$ nas lesões graves ${ }^{19,20}$. As mortes são resultado de hemorragia incontrolada, complicações infecciosas pós operatórias, extensão das lesões associadas e disfunção orgânica de múltiplos sistemas ${ }^{21-23}$. Segundo estudo prospectivo de 164 casos de trauma hepático no Hospital de Emergências José Casimiro Ulloa em Lima, Peru ${ }^{24}$, a relação entre os graus de lesão hepática e a mortalidade foram: Grau I $(5,49 \%)$ com $0 \%$ de mortalidade; Grau II (59,15\%) com 4,1\% de mortalidade; Grau III (22,56\%) com $5 \%$ de mortalidade; Grau IV (7,32\%) com 58\% de mortalidade 
Tabela 1 - Classificação da AAST para lesão hepática.

\begin{tabular}{|c|c|c|}
\hline Grau & Lesão & Características ao exame tomográfico \\
\hline \multirow[t]{2}{*}{ I } & Hematoma & Subcapsular $<10 \%$ da área de superfície. \\
\hline & Laceração & Ruptura capsular $<1 \mathrm{~cm}$ de profundidade no parênquima. \\
\hline \multirow[t]{2}{*}{ II } & Hematoma & Subcapsular, 10 a $50 \%$ da área de superfície. Intraparenquimatoso, $<2 \mathrm{~cm}$ de diâmetro. \\
\hline & Laceração & $1 \mathrm{a} 3 \mathrm{~cm}$ de profundidade no parênquima, $<10 \mathrm{~cm}$ de extenção. \\
\hline \multirow[t]{2}{*}{ III } & Hematoma & $\begin{array}{l}\text { Subcapsular, > que } 50 \% \text { da área de superfície ou em expansão. Ruptura subcapsular ou hematoma } \\
\text { parenquimatoso. Hematoma intraparenquimatoso }>2 \mathrm{~cm} \text { ou em expansão. }\end{array}$ \\
\hline & Laceração & Maior que $3 \mathrm{~cm}$ de profundidade. \\
\hline IV & Laceração & $\begin{array}{l}\text { Dilaceração do parênquima envolvendo } 25-75 \% \text { do lobo hepático ou 1-3 segmentos de Coinaud ou } \\
\text { mesmo lobo. }\end{array}$ \\
\hline \multirow[t]{2}{*}{$\mathrm{V}$} & Laceração & Dilaceração do parênquima > 75\% do lobo hepático ou > 3 segmentos de Coinaud no mesmo lobo. \\
\hline & Vascular & Lesões de veias justahepáticas ou veias hepáticas/veia cava retrohepática. \\
\hline VI & Vascular & Avulsão hepática. \\
\hline
\end{tabular}

The American Association for the Surgery of Trauma.

e Grau V (5,49\%) com 100\% de mortalidade, o que condiz com os resultados encontrados neste trabalho.

O manejo inicial do paciente traumatizado deve seguir os princípios do Advanced Trauma Life Support (ATLS) ${ }^{25}$. O desenvolvimento do atendimento pré-hospitalar, o transporte rápido e da reanimação adequada das vítimas de trauma, têm aumentado a taxa de sobrevida dos pacientes gravemente traumatizados que há algum tempo vinham à êxito letal na cena do acidente ou no caminho até o hospital ${ }^{14}$.

O diagnóstico das lesões intra-abdominais pode ser feito rapidamente quando há instabilidade hemodinâmica, sinais de peritonite, distensão abdominal em evolução e lesões penetrantes. Entretanto, frequentemente há necessidade de métodos diagnósticos complementares. OFAST (Focused Assesment With Sonography In trauma ) é um exame de alta sensibilidade e amplamente usado para detectar hemoperitônio em pacientes instáveis. O lavado peritoneal é útil quando o paciente tem alteração sensorial e está hemodinamicamente instável, e na instituição não há ultra-som e tomografia disponíveis. Por outro lado, quando o paciente está estável hemodinamicamente a tomografia computadorizada com triplo contraste é o método de escolha em pacientes com trauma abdominal contuso ${ }^{26}$. Os grandes avanços da TC estão na habilidade de determinar a extensão da lesão hepática, documentar a presença de hemorragia ativa e detectar lesões associadas. Porem é importante enfatizar que a decisão em proceder com tratamento cirúrgico em pacientes com lesões hepáticas está indicada por sua condição hemodinâmica e não pelos achados a tomografia ${ }^{5}$.

O tratamento conservador do trauma hepático contuso apresenta vantagens em relação ao operatório a saber: há a menor necessidade de transfusão sanguínea, menor ocorrência de sepse intra-abdominal ${ }^{27}$ e menor mortalidade ${ }^{28}$. Para tanto o paciente precisa ser mantido em unidade de terapia intensiva, não pode exibir sinais de peritonite e deve estar hemodinamicamente estável sem necessidade de volume significante de transfusão sanguínea. Lesões de Grau IV e V associadas com hemoperitônio não são contra-indicações, mas estão associadas a maiores taxas de morbidade e falha (14 a $52 \%$ ) do que nos Graus I a III ( 3 a 7,5\% $)^{29,30}$. Embora o tratamento não operatório de lesões penetrantes tenha sido descrito em pacientes altamente selecionados ele não é considerado a terapia padrão e a sua aplicação ainda é objeto de intenso debate ${ }^{31}$.

A penetração na cavidade abdominal é a indicação padrão para laparotomia exploradora ${ }^{26}$. Muitos procedimentos podem ser realizados como: simples suturas, ressecções associadas a desbridamentos ou mesmo, em lesões ainda mais graves, o empacotamento ${ }^{14}$. O tratamento cirúrgico de pequenas lesões hepáticas geralmente pode ser feito com cauterização ou pequenas suturas. As lesões mais graves, a despeito dos avanços tecnológicos, constituem ainda um desafio aos cirurgiões.

\begin{abstract}
Background: We analyze the epidemiological characteristics related to hepatic trauma, and we do a brief review of the different types in diagnosis and management. Methods: A retrospective study from February 2002 to March 2007. One hundred fifty four patients were admitted at Cajuru University Hospital with hepatic trauma. Results: We found that $90.26 \%$ of hepatic trauma victims were male with average age of 26.28 years old. Hepatic no blunt traumas were $72.73 \%$. Among these, $55.54 \%$ were firearm traumas and $16.88 \%$ were caused by stab wounds. Blunt traumas were responsible by automobile collisions in $73.81 \%$, and $26.49 \%$ were by other mechanisms. Most patients arrived at the hospital between 12:00 A.M. to 12:00 P.M. The average blood pressure was 117.6/72.3 mmHg, the average cardiac frequency was $99.03 \mathrm{bpm}$ and the Glasgow average was 13.6. In 60.43\% of the cases, the time gap between admission and surgery took less than 2 hours. The most frequent lesions were related to a second degree followed by $3 \mathrm{rd}$ and 4 th degree lesions reaching $88.3 \%$ of the cases. Associated lesions treated surgically were found in more than 75\% of the cases. The average ISS was 15.09, 19.85, $27.83,35.47$, and $40.93 \%$. The survival rate was 100, 88.88, 81.85, 48.88, and 22.23\% in 1st, 2nd, 3rd, 4th, and 5th degree lesions, respectively. Conclusion: The epidemiological data reflect modern society violence, which is translated by the increasing complexity in lesions found, and it has been a challenge for the surgeon to choose the best therapeutic method.
\end{abstract}

Key words: Liver; Abdominal injuries; Wounds and injuries/epidemiology. 


\section{REFERÊNCIAS}

1. Lee SK, Carrillo EH. Advances and changes in the management of liver injuries. Am Surg. 2007;73(3):201-6.

2. Velho AV, Ostermann RAB, Dacanal FM, Bayer LR. Análise dos fatores preditivos de complicações após trauma hepático penetrante. Rev Col Bras Cir.1999;26(2):97-101.

3. Croce MA, Fabian TC, Menke PG, Waddle-Smith L, Minard G, Kudsk KA, et al. Nonoperative management of blunt hepatic trauma is the treatment of choice for hemodynamically stable patients. Results of a prospective trial. Ann Surg. 1995;221(6):744-53; discussion 753-5.

4. Gür S, Orsel A, Atahan K, Hökmez A, Tarcan E. Surgical treatment of liver trauma (analysis of 244 patients). Hepatogastroenterology. 2003;50(54):2109-11.

5. Carrillo EH, Platz A, Miller FB, Richardson JD, Polk HC Jr. Non-operative management of blunt hepatic trauma. $\mathrm{Br} \mathrm{J}$ Surg.1998;85(9):461-8.

6. Scollay JM, Beard D, Smith R, McKeown D, Garden OJ, Parks R. Eleven years of liver trauma: the Scottish experience. World J Surg. 2005;29(6):744-9.

7. Schweizer W, Tanner S, Baer HU, Lerut J, Huber A, Gertsch P, Blumgart LH. Management of traumatic liver injuries. Br J Surg. 1993;80(1):86-8.

8. Matsch $\mathrm{T}$, Begquist $\mathrm{D}$, Hedelin $\mathrm{M}$, Findblack B. Leberverletzungen nachstumpfem Bauchtrauma. Unfallchirurgie. 1982;85:524-8.

9. Watson CJ, Calne RY, Padhani AR, Dixon AK. Surgical restraint in the management of liver trauma. Br J Surg. 1991;78(9):10715.

10. Talving P, Beckman M, Häggmark T, Iselius L. Epidemiology of liver injuries. Scand J Surg. 2003;92(3):192-4.

11. Krige JE, Bornman PC, Terblanche J. Liver trauma in 446 patients. S Afr J Surg. 1997;35(1):10-5.

12. Feliciano DV, Mattox KL, Jordan GL Jr, Burch JM, Bitondo CG, Cruse PA. Management of 1000 consecutive cases of hepatic trauma (1979-1984). Ann Surg. 1986;204(4):438-45.

13. Von Bahten LC, Nicoluzzi JE, Olandoski M, Pantanali CA, Silva RFK. Trauma abdominal fechado: análise dos pacientes vítimas de trauma hepático em um Hospital Universitário de Curitiba. Rev Col Bras Cir. 2005;32(6):316-20.

14. Stalhschmidt CMM, Formighieri B, Lubachevski FB. Controle de danos no trauma abdominal e lesões associadas: experiência de cinco anos em um serviço de emergência. Rev Col Bras Cir. 2006;33(4):215-9

15. Hurtuk, MB, Reed LR 2 ${ }^{\text {nd }}$, Esposito TJ, Davis KA, Luchette FA. Trauma surgeons practice what they preach: the NTDB story on solid organ injury management. J Trauma. 2006;61(2):243-54; discussion 254-5.

16. Matthes G, Stengel D, Seifert J, Rademacher G, Mutze S, Ekkernkamp A. Blunt liver injuries in polytrauma: results from a cohort study with the regular use of a whole-body helical computed tomography. World J Surg. 2003;27(10):1124-30.

17. Pachter HL, Knudson MM, Esrig B, Ross S, Hoyt D, Cogbill T, et al. Status of nonoperative management of blunt hepatic injuries in 1995: a multicenter experience with 404 patients. J Trauma. 1996;40(1):31-8.
18. Moore EE, Cogbill TH, Jurkovitch GJ, Shackford SR, Malangoni MA, Champion HR. Organ injury scaling: spleen and liver (1994 revision). J Trauma. 1995;38(3):323-4.

19. Trunkey DD. Hepatic trauma: contemporary management. Surg Clin North Am. 2004;84(2):437-50.

20. Anderson IB, Al Saghier M, Kneteman NM, Bigam DL. Liver trauma: management of devascularization injuries. J Trauma. 2004;57(5):1099-104.

21. Duane TM, Como JJ, Bochicchio GV, Scalea TM . Reevaluating the management and outcomes of severe blunt liver injury. J Trauma. 2004;57(3):494-500.

22. Christmas AB, Wilson AK, Manning B, Franklin GA, Miller FB, Richardson JD, Rodriguez JL. Selective management of blunt hepatic injuries including nonoperative management is a safe and effective strategy. Surgery. 2OO5;138(4):606-10; discussion 6101.

23. Cogbill TH, Moore EE, Jurkovich GJ, Feliciano DV, Morris JA, Mucha P. Severe hepatic trauma: a multi-center experience with 1,335 liver injuries. J Trauma. 1988;28(10):1433-8.

24. Duránd López CA, Delgado V. B. Trauma hepático. Rev Gastroenterol Peru. 2001;21(2):115-22.

25. American College of Surgeons. Advanced trauma life support. $7^{\text {th }}$ ed. Chicago: American College of Surgeons; 2004.

26. Stracieri LD, Scarpelini S. Trauma hepático. Acta Cir Bras. 2006;21(Supl.1):85-8.

27. Veroux M, Cillo U, Brolese A, Veroux P, Madia C, Fiamingo P, et al. Blunt liver injury: from non-operative management to liver transplantation. Injury. 2003;34(3):181-6.

28. Hsieh CH, Chen RJ, Fang JF, Lin BC, Hsu YP, Kao JL, et al. Liver abscess after non-operative management of blunt liver injury. Langenbecks Arch Surg. 2003;387(9-10):343-7. Epub 2002 Dec 12.

29. Malhotra AK, Fabian TC, Croce MA, Gavin TJ, Kudsk KA, Minard G, Pritchard FE. Blunt hepatic injury: a paradigm shift from operative to nonoperative management in the 1990s. Ann Surg. 2000;231(6):804-13.

30. Kozar RA, Moore FA, Cothren CC, Moore EE, Sena M, Bulger $\mathrm{EM}$, et al. Risk factors for hepatic morbidity following nonoperative management: multicenter study. Arch Surg. 2006;141(5):451-8; discussion 458-9.

31. Omoshoro-Jones JA, Nicol AJ, Navsaria PH, Zellweger R, Krige JE, Kahn DH. Selective non-operative management of liver gunshot injuries. Br J Surg. 2005;92(7):890-5.

Como citar este artigo:

Stalhschmidt CM, Formighieri B, Marcon DM, Takejima AL, Soares LGS. Trauma hepático: epidemiologia de cinco anos em um serviço de emergência. Rev Col Bras Cir. [periódico na Internet] 2008; 35(4). Disponível em URL: http://www.scielo.br/rcbc

Endereço para correspondência:

Carla Martinez Menini Stalhschmidt

Rua Peru, 173 - casa 5

Bacacheri

82510-140 - Curitiba - PR

E-mail: carlamenini@uol.com.br 